\title{
Increased circulating T-helper 22 cells in patients with dilated cardiomyopathy
}

\author{
QING KONG, XIAOMO LI, WEIFENG WU, FAN YANG, YANLI LIU, WENYIN LAI \\ XIAOFEN PAN, MENGSHA GAO and YIMIN XUE
}

\begin{abstract}
Department of Cardiology, First Affiliated Hospital of Guangxi Medical University, Guangxi Cardiovascular Institute, Nanning, Guangxi 530021, P.R. China
\end{abstract}

Received August 2, 2013; Accepted March 6, 2014

DOI: $10.3892 / \mathrm{mmr} .2014 .2146$

\begin{abstract}
Recently, the newly determined interleukin (IL)-22-producing T-helper (Th) 22 cell has been implicated to be involved in the pathogenesis of autoimmune diseases. However, its role in the pathogenesis of dilated cardiomyopathy (DCM) has yet to be elucidated. A total of 30 patients with DCM and 30 healthy controls were enrolled in the present study. The levels of Th22, Th17 and Th1 cells in the peripheral blood were analyzed by flow cytometry. Levels of plasma IL-22 and autoantibody adenine nucleotide translocator (ANT) were assessed using the ELISA. The key transcription factor of Th22, aryl hydrocarbon receptor (AHR), was assessed using quantitative polymerase chain reaction. Additionally, clinical data on the brain natriuretic peptide (BNP), C-reactive protein (CRP) and erythrocyte sedimentation rate (ESR) were collected. In comparison with those in the control group, significantly elevated levels of Th22, Th17 and Th1 cells were detected in patients with DCM (all $\mathrm{P}<0.01$ ). Similarly, elevated mRNA levels of peripheral AHR were detected in patients with DCM. The percentage of Th22 cells was higher in ANT-positive compared with ANT-negative patients with DCM. The levels of BNP and CRP, but not ESR, showed a significant positive correlation with those of Th22 cells. With regard to the concentrations of plasma IL-22, no statistical difference was found between patients with DCM and the healthy controls, nor did it demonstrate a statistical correlation with the percentage of
\end{abstract}

Correspondence to: Professor Weifeng Wu, Department of Cardiology, First Affiliated Hospital of Guangxi Medical University, Guangxi Cardiovascular Institute, 22 Shuangyong Road, Nanning, Guangxi 530021, P.R. China

E-mail:wucna65@163.com

Abbreviations: qPCR, quantitative polymerase chain reaction; DCM, dilated cardiomyopathy; IL-22, interleukin-22; ANT, adenine nucleotide translocator; BNP, brain natriuretic peptide; ESR, erythrocyte sedimentation rate; $\mathrm{CRP}, \mathrm{C}$-reactive protein; $\mathrm{VMC}$, viral myocarditis

Key words: T-helper 22 cells, interleukin 22, dilated cardiomyopathy, immunity, inflammation
Th22 cells. In conclusion, the present study showed that patients with DCM, particularly those of the ANT autoantibody positive subjects, exhibit elevated levels of peripheral Th22 cells, indicating that a Th22 immune response may be implicated in the pathogenesis of DCM.

\section{Introduction}

Dilated cardiomyopathy (DCM), the most common of the cardiomyopathies and is characterized by the progressive deterioration of myocardial contractile function and ventricular dilation $(1,2)$. The five-year survival rate following diagnosis is $50 \%$, as patients often develop progressive congestive heart failure and complications, including thromboembolic conditions and arrhythmias $(2,3)$. Although it has been described that the dysregulation of T-cells, including $\mathrm{T}$ helper (Th) 1 cells, Th2, Th17 and regulatory T-cells, have a critical role in the autoimmune response of DCM (4-7), with the discovery of the anti-heart antibodies, including the autoantibody against the adenine nucleotide translocator (ANT) (8), the pathophysiology of DCM remains to be fully elucidated.

Th22 cells are a novel subset of T cells clearly separated from other known Th cells. Th22 is distinct from interleukin (IL)-17-secreting and retinoic acid receptor-related orphan receptor $\gamma$ t-regulated Th17, interferon (IFN)- $\gamma$-secreting and T-bet-regulated Th1 or IL-4-secreting and trans-acting T-cell-specific transcription factor GATA-3-regulated Th2 cells. Th22 is characterized by producing abundant cytokine IL-22 and is regulated by the key transcription factor aryl hydrocarbon receptor (AHR) and other cytokines, including tumor necrosis factor- $\alpha$ and IL-6 $(9,10)$. Excluding the chemokine receptor $\mathrm{C}-\mathrm{C}$ chemokine receptor (CCR) type 10, Th22 shares common surface markers with Th17 cells, including CCR6 and CCR4. In previous years, studies have focused on the participation of Th22 cells in various human inflammatory disorders and immune-mediated tissue injury, including rheumatoid arthritis, Crohn's disease and systemic sclerosis. The elevation of circulating Th22 cells was positively correlated with the levels of autoimmune antibodies, Th17, Th1 cells and the severity of several diseases (11-15). Up to date, the involvement of IL-22 in DCM has remained to be elucidated and no data exist with regard to Th22 cells and their association with Th17 or Th1 in patients with DCM. 
In the present study, the percentages of peripheral Th22, Th17 and Th1, the expression of AHR in peripheral blood mononuclear cells (PBMCs) as well as the levels of IL-22, ANT, brain natriuretic peptide (BNP), erythrocyte sedimentation rate (ESR) and C-reactive protein (CRP) in the peripheral blood of patients with DCM were measured, and their relevance was evaluated.

\section{Materials and methods}

Patients and controls. The study included 60 individuals, comprising 30 cases with primary DCM and 30 normal individuals (controls) and was conducted between December 2010 and February 2013. Primary DCM was diagnosed based on the guidelines of the World Health Organization (1). Accordingly, various secondary causes of heart failure, including coronary artery disease and valvular diseases, were excluded in order to diminish the potential confounding effects of etiological heterogeneity of heart failure. A control group of normal individuals, defined as asymptomatic age- and gender-matched individuals with normal electrocardiograms and echocardiograms, was included. A normal echocardiogram excluded the diagnosis of DCM in the control individuals. Patients who were receiving anti-inflammatory or immunosuppressive medications, recently treated with antibiotics and other underlying acute or chronic diseases, were all excluded from the present study. To reduce the potential confounding effects of diabetes mellitus, those who had this disease were excluded. The present study was performed in accordance with protocols approved by the Guangxi Medical University Ethics Committee (Guangxi, China) and the participants gave informed consent. The clinical data, including ESR and CRP were also collected. The characteristics of the study subjects are summarized in Table I.

Isolation and stimulation of PBMCs. Peripheral blood was harvested with $50 \mathrm{U} / \mathrm{ml}$ heparin and diluted with an equal volume of phosphate-buffered saline. PBMCs were isolated using Ficoll-Plaque (Solarbio Science \& Technology, Beijing, China) by gradient centrifugation at $300 \mathrm{x}$ g and cells were resuspended with a final concentration of $2 \times 10^{6}$ in complete RPMI-1640 (Solarbio Science \& Technology) supplemented with $100 \mathrm{U} / \mathrm{ml}$ penicillin and $100 \mu \mathrm{g} / \mathrm{ml}$ streptomycin, followed by incubation at $37^{\circ} \mathrm{C}$ with $5 \% \mathrm{CO}_{2}$ for $5 \mathrm{~h}$ in the presence of $25 \mathrm{ng} / \mathrm{ml}$ phorbol myristate acetate (PMA) $1 \mu \mathrm{g} / \mathrm{ml}$ ionomycin (both from Sigma-Aldrich, St. Louis, MO, USA) and Golgi Plug (1 $\mu \mathrm{l} / 10^{6}$ cells; BD Biosciences, San Diego, CA, USA).

Flow cytometric analysis. The expression of markers on $\mathrm{T}$ cells from blood were determined by flow cytometry following surface or intracellular staining with anti-human-specific antibodies. Briefly, following incubation, the PBMCs were stained with fluorescein isothyocyanate-conjugated anti-CD4 monoclonal antibodies (cat no. 555346; BD Biosciences) at room temperature in the dark for $30 \mathrm{~min}$. Next, the cells were stained with allophycocianin-conjugated anti-IFN- $\gamma$ (cat no. 554702; BD Biosciences), peridinin chlorophyll-CY5.5-conjugated anti-IL-17A (cat no. 560799; BD Biosciences) and phycoerythrin-conjugated anti-IL-22 monoclonal antibodies (cat no. 12-7229-42; eBioscience, San Diego, CA, USA)
Table I. Characteristics of patients with DCM and control (mean \pm standard deviation)

\begin{tabular}{lcc}
\hline Characteristics & DCM & Control \\
\hline Patients, $\mathrm{n}$ & 30 & 30 \\
Age, years & $51.6 \pm 7.1$ & $49.8 \pm 6.7$ \\
Male/female (n) & $8 / 22$ & $8 / 22$ \\
Heart rate, beats/min & $74.3 \pm 12.6$ & $77.1 \pm 14.1$ \\
Systolic BP, mmHg & $115.2 \pm 14.6$ & $126.1 \pm 17.5$ \\
Diastolic BP, mmHg & $68.2 \pm 8.4$ & $74.7 \pm 11.3$ \\
Functional class (NYHA) & & \\
I & 4 & 30 \\
II & 6 & 0 \\
III & 9 & 0 \\
IV & 11 & 0 \\
LVEF, $\%$ & $23.2 \pm 7.2$ & $62.5 \pm 8.9$ \\
LVEDD, mm & $63.8 \pm 12.1$ & $46.7 \pm 6.8$ \\
LVESD, mm & $52.1 \pm 12.1$ & $33.4 \pm 6.4$ \\
Medications & & \\
$\beta$-blockers & 32 & 0 \\
ACE inhibitors & 21 & 1 \\
ATR blockers & 3 & 0 \\
Aldosterone receptor blockers & 10 & 0 \\
Furosemide & 21 & 0 \\
Digoxin & 12 & 0 \\
\hline
\end{tabular}

Class I, no cardiac symptoms even with activity; class II, cardiac symptoms lead to some difficulties with usual activity; class III, cardiac symptoms make patient unable to perform usual physical activity; or class IV, cardiac symptoms present even at rest. DCM, dilated cardiomyopathy; BP, blood pressure; NYHA, New York Heart Association; LVEF, left ventricular ejection fraction; LVEDD, left ventricular end-diastolic diameter; LVESD, left ventricular end-systolic diameter; ACE, angiotensin-1 converting enzyme-1; ATR, angiotensin II receptor.

following fixation and permeabilization. Isotype controls (BD Bioscience) were given to enable correct compensation and confirm the antibody specificity. Stained cells were analyzed by flow cytometric analysis using a FACScan cytometer equipped with CellQuest software (BD Biosciences). Pure Th22 cells were defined as IL-22+IL-17- IFN- $\gamma^{-} \mathrm{CD}^{+}{ }^{+}$. The Th17 cells were identified as those that were $\mathrm{CD} 4^{+} \mathrm{IL}-17^{+}$, and $\mathrm{Th} 1$ cells were $\mathrm{CD} 4{ }^{+} \mathrm{IFN}-\gamma^{+}$.

AHR mRNA expression by quantitative-polymerase chain reaction ( $q P C R$ ). TRIzol reagent (Invitrogen, Carlsbad, CA, USA) was used to isolate the total RNA of PBMCs. The RNA was transcribed into cDNA with a reverse transcription kit (Ferma, Mountain View, CA, USA) according to the manufacturer's instructions. qPCR was performed for AHR and the housekeeping gene $\beta$-actin on an ABI 7500 Real-Time PCR System (Applied Biosystems, Grand Island, NY, USA) using $\mathrm{SYBR}^{\circledR}$ Green. The PCR reactions were cycled 40 times following initial denaturation at $94^{\circ} \mathrm{C}$ for 3 min with 
the following parameters: Denaturation at $94^{\circ} \mathrm{C}$ for $30 \mathrm{sec}$, annealing at $60^{\circ} \mathrm{C}$ for $30 \mathrm{sec}$ and extension at $72^{\circ} \mathrm{C}$ for $60 \mathrm{sec}$. The primers used were as follows: AHR forward, 5'-ACT CCA CTT CAG CCA CCA TC-3' and reverse, 3'-ATG GGA CTC GGC ACA ATA AA-5'; $\beta$-actin forward, 5'-GCA AGC AGG AGT ATG ACG AG-3' and reverse, 3'-CAA ATA AAG CCA TGC CAA TC-5'. The relative gene expression of AHR was normalized to the level of $\beta$-actin transcripts and quantified by the $\Delta \Delta \mathrm{Ct}$ method using 7500 System Sequence Detection software (Applied Biosystems, Foster City, CA, USA). All experiments were conducted in triplicate.

IL-22 and BNP assessment by ELISA. Serum was obtained from all subjects by centrifugation at $200 \mathrm{x}$ g and stored at $-80^{\circ} \mathrm{C}$ for the determination of cytokine levels. Serum IL-22 levels were determined with a quantitative sandwich enzyme immunoassay technique following the manufacturer's instructions (cat no. BMS2047; eBioscience). The levels of BNP were measured using ELISA (cat no. CSB-E07970h; Cusabio, Wuhan, China). The minimum detectable concentration was $5 \mathrm{pg} / \mathrm{ml}$ for IL-22 and $23 \mathrm{pg} / \mathrm{ml}$ for BNP, respectively. All the samples were assayed in duplicate.

ANT assessment by ELISA. For the qualitative determination of human ANT autoantibody concentrations in serum, an ANT autoantibody ELISA kit was used according to manufacturer's instructions (cat no. CSB-EQ027458HU; Cusabio, Wuhan, China). In order to calculate the valence of the human ANT autoantibody, the sample well was compared with the control. A cutoff value was defined as the average negative control value plus 0.2 . An optical density (OD) sample $<$ the cutoff value was negative, while an OD sample $\geq$ the cut off value was positive.

Statistical analysis. Quantitative variables were expressed as the mean \pm standard deviation. Differences between two groups were determined using the Newman-Keuls multiple comparison test (q-test). The Pearson's or Spearman's correlation test was used for correlation analysis depending on the data distribution. Qualitative variables of ANT were expressed as percentages and differences in ANT distribution between groups were obtained using the $\chi^{2}$ test. Analysis was performed using SPSS version 17.0 (SPSS, Inc., Chicago, IL, USA). $\mathrm{P}<0.05$ was considered to indicate a statistically significant difference.

\section{Results}

Elevated circulating Th22, Th17 and Th1 frequencies in DCM. The frequencies of Th22, Th17 and Th1 based on cytokine patterns following in vitro activation by phorbol myristate acetate, ionomycin and monensin were analyzed in short-term cultures. Pure Th22 was defined as CD4 $4^{+}$IFN- $\gamma^{-}$IL-17-IL-22 ${ }^{+}$ T cells to exclude Th1 or Th17 cells. Th17 and Th1 cells were identified as $\mathrm{CD} 4{ }^{+} \mathrm{IL}-17^{+} \mathrm{T}$ cells and IFN- $\gamma^{+} \mathrm{CD} 4^{+} \mathrm{T}$ cells, respectively. Representative plots of the proportion of $\mathrm{Th} 22$, Th17 and Th1 cells in a representative patient with DCM and a healthy control are shown in Fig. 1A-C. As shown in Fig. 2A, the frequencies of Th1 cells were significantly elevated in patients with DCM as compared with the healthy donors $(5.29 \pm 1.82$ vs. $2.81 \pm 0.97 \%$; $\mathrm{P}<0.01)$. The proportions
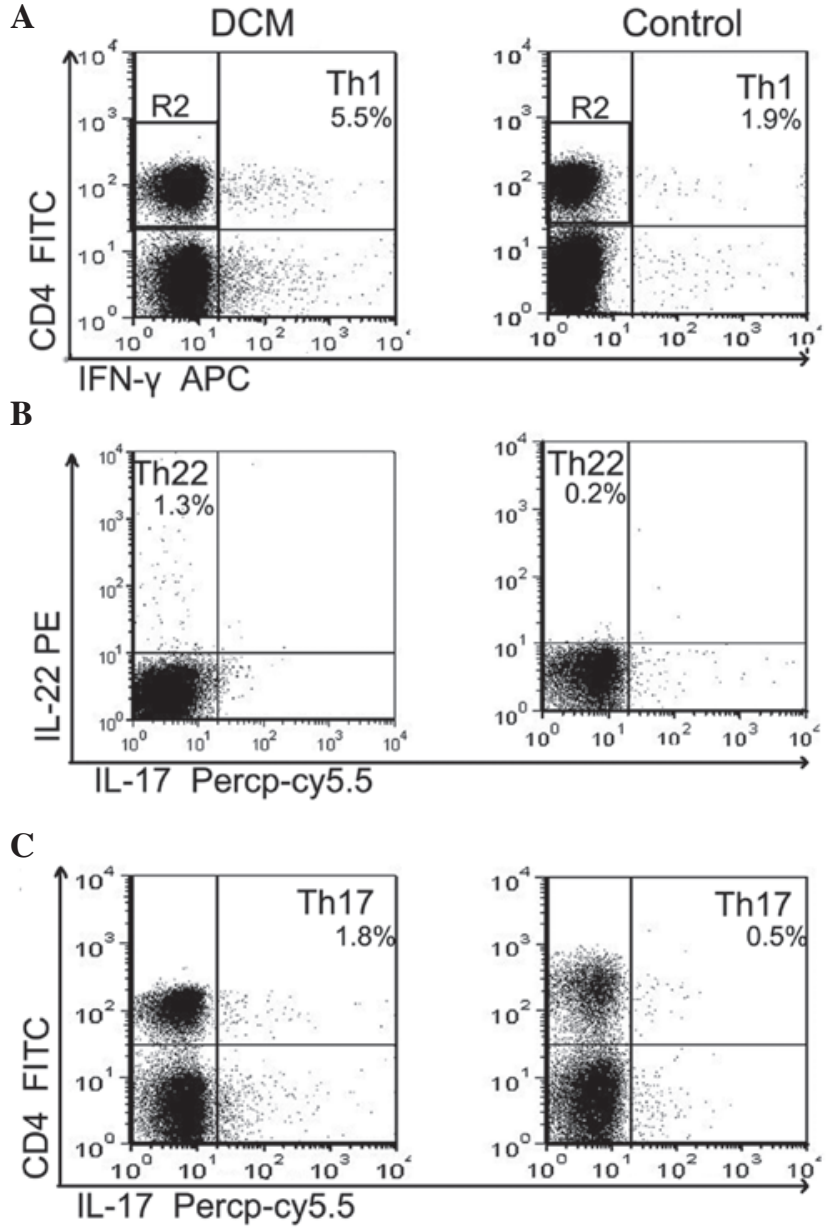

Figure 1. Circulating Th22, Th17 and Th1 cells in patients with DCM and healthy controls. Heparinized peripheral whole blood from patients with $\operatorname{DCM}(n=30)$ and healthy donors $(n=30)$ were stimulated with phorbol myristate acetate, ionomycin and monensin for $4 \mathrm{~h}$, and then stained with labeled antibodies for fluorescence-activated cell-sorting analysis (A) Representative flow cytometry dot plots of (A) circulating Th1 cells $\left(\mathrm{CD} 4^{+} \mathrm{IFN}-\gamma^{+}\right)$, (B) peripheral Th22 cells $\left(\mathrm{CD} 4^{+} \mathrm{IL}-22^{+} \mathrm{IFN}-\gamma^{-} \mathrm{IL}^{-17^{-}}\right)$and (C) circulating Th17 cells $\left(\mathrm{CD} 4^{+} \mathrm{IL}-17^{+}\right)$from patients with DCM and normal controls. The numbers in the plots indicate relative percentages per quadrant. DCM, dilated cardiomyopathy; Th, T-helper; IL, interleukin; FITC, fluorescein isothiocyanate; PR, phycoerythrin; Percp; peridinin chlorophyll.

of the peripheral Th22 cells were significant higher in the DCM group $(0.91 \pm 0.34 \%)$ in comparison with the control group $(0.26 \pm 0.08 \%$; $\mathrm{P}<0.01)$ (Fig. $2 \mathrm{~B})$. Similarly, the percentages of the Th17 cells were profoundly increased in the DCM group $(2.27 \pm 0.59 \%)$ as compared with the control group $(0.27 \pm 0.07 \%$; $<<0.01)$ (Fig. 2C).

Positive correlation between Th22 and Th17 cells in DCM. Correlations between the frequencies of Th22, Th17 and Th1 cells in patients with DCM were examined. There was a significant positive correlation between numbers of Th22 and Th17 cells, as shown in Fig. 2D ( $\mathrm{r}=0.676$ and $\mathrm{P}<0.001)$. However, the correlation analysis between the percentages of Th1 and Th22 cells showed no significant association in patients with DCM (r=0.326 and $\mathrm{P}=0.052)$ (Fig. 2E).

Enhanced mRNA expression of peripheral AHR in DCM. The mRNA expression of AHR, the key transcription factor directing Th22 lineage commitment, was determined by 

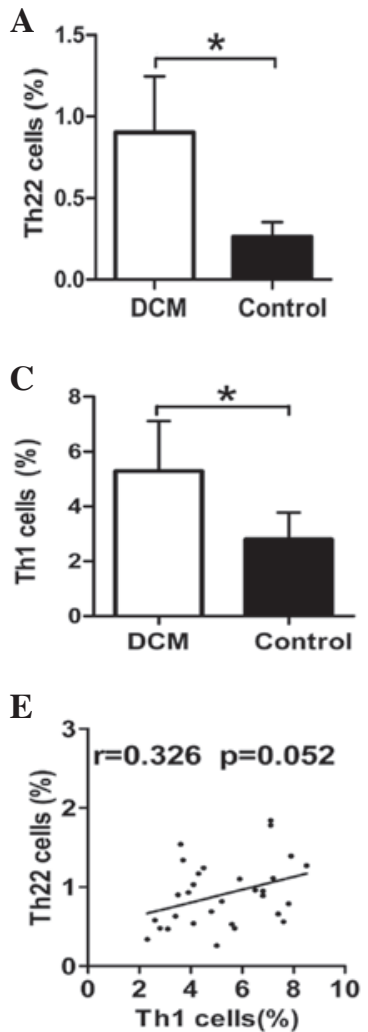
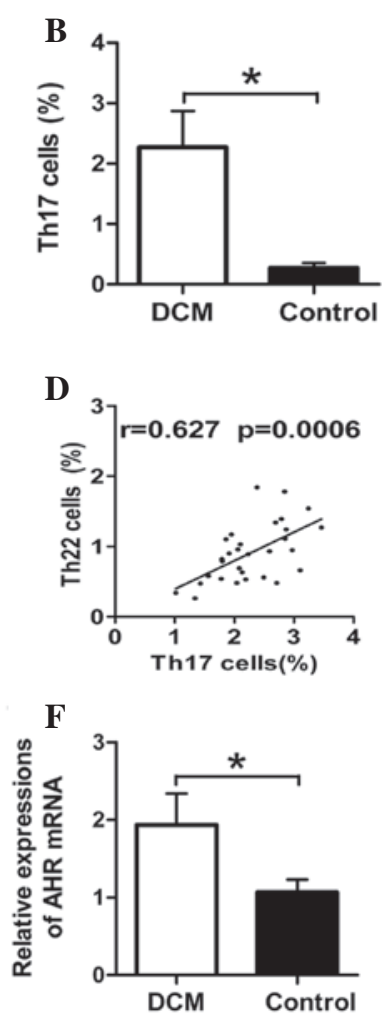

Figure 2. Increased circulating percentages of Th22, Th17 and Th1 cells in DCM. (A) Results of statistical analysis for the alterations of Th22 cells measured by fluorescence-activated cell sorting analysis. Increased frequencies of Th22 cells were found in the DCM group. (B and C) Elevated percentages of Th17 and Th1 cells were observed in the DCM group. (D and E) Correlation analysis of Th22 and Th17, Th22 and Th1 cells in the DCM group. Each point represents an individual. A significant positive correlation was found between Th22 and Th17 cells, but not between the Th22 and Th1 cells. (F) Relative expression of AHR mRNA, the key transcription factor of Th22 cells detected by quantitative polymerase chain reaction, was higher in the DCM group. ${ }^{*} \mathrm{P}<0.01$. Bars represent the standard deviation. DCM, dilated cardiomyopathy; AHR, aryl hydrocarbon receptor; Th, T-helper.

qPCR to further confirm the results of the present study obtained from flow cytometric analysis. Consistent with the higher proportion of Th22 cells in patients with DCM, the data demonstrated that levels of the relative AHR transcript were notably higher in the PBMCs of patients with DCM as compared with those in the healthy control $(1.93 \pm 0.4 \mathrm{vs}$. $1.06 \pm 0.16 ; \mathrm{P}<0.01$ ) (Fig. 2F).

Plasma levels of IL-22. Plasma levels of IL-22 were evaluated by ELISA. Of note, the expression of IL-22 did not differ between patients with DCM and healthy controls (19.78 \pm 5.9 vs. $21.3 \pm 5.4 \mathrm{pg} / \mathrm{ml} ; \mathrm{P}=0.303$ ) (Fig. $3 \mathrm{~A}$ ). Furthermore, no significant correlation was identified between Th22 cells and IL-22 levels ( $\mathrm{r}=0.345$ and $\mathrm{P}=0.061$; Fig. 3B), nor between Th17 cells and IL-22 ( $\mathrm{r}=0.235$ and $\mathrm{P}=0.211$; Fig. $3 \mathrm{C}$ ). As shown in Fig. 3D, the peripheral Th1 cells did not show a statistical correlation with circulating levels of IL-22 $(r=0.221$ and $\mathrm{P}=0.241)$.

Th22 cells but not IL-22 levels are increased in ANT antibody-positive patients with DCM. The percentages of ANT-positive individuals among patients with DCM and healthy controls were 40 and $6.7 \%$, respectively $(\mathrm{P}<0.05)$.
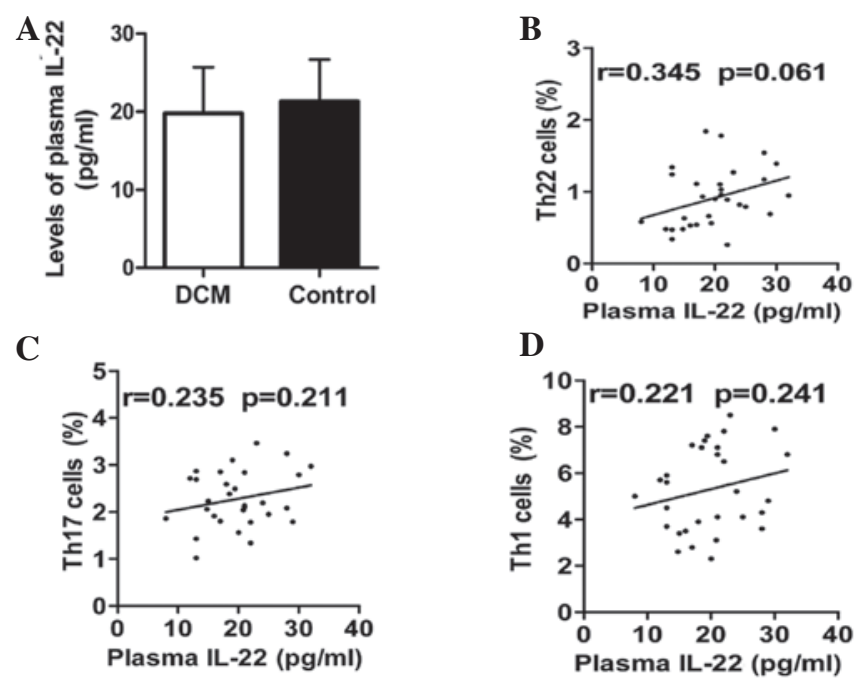

D

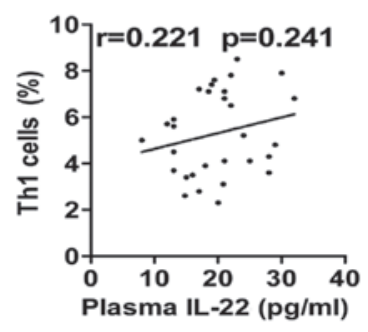

Figure 3. Expression of IL-22 in patients with DCM and healthy controls. (A) Concentrations of IL-22 plasma from patients with DCM and healthy controls were detected by ELISA. Levels of IL-22 were comparable between these two groups. (B-D) Correlation analysis of peripheral (B) Th22 cells and IL-22, (C) Th17 cells and IL-22 and (D) Th1 cells and IL-22 in the DCM group. No significant correlation was observed. Each point represents an individual. Bars represent the standard deviation. IL, interleukin; DCM, dilated cardiomyopathy.
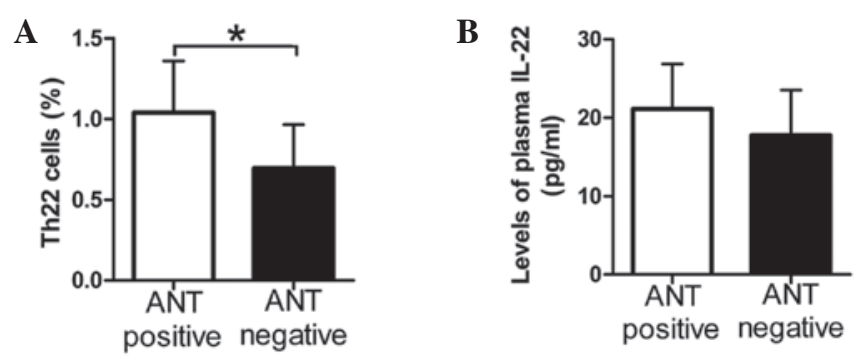

Figure 4. Th22 cells but not IL-22 levels are elevated in ANT antibody-positive patients with DCM. Peripheral Th22 cells and plasma IL-22 levels were compared between ANT-positive and -negative patients with DCM. (A) The percentages of circulating Th22 cells from ANT-positive patients with DCM were significantly higher compared with those from ANT-negative patients. (B) With regard to plasma IL-22, there was no significant difference between ANT-positive and -negative patients with DCM. ${ }^{*} \mathrm{P}<0.01$. Bars represent the standard deviation. IL, interleukin; ANT, adenine nucleotide translocator; DCM, dilated cardiomyopathy; Th, T-helper.

It was investigated whether the levels of Th22 cells and IL-22 in the DCM group $(n=30)$ demonstrated a difference between ANT-positive and -negative patients. Compared with ANT-negative patients with DCM, levels of circulating Th22 cells were increased in ANT-positive patients with DCM ( $1.04 \pm 0.32$ vs. $0.69 \pm 0.26 \%$; $\mathrm{P}=0.005$; Fig. 4A). However, the levels of plasma IL-22 did not differ between ANT antibody-positive and -negative individuals (21.11 \pm 5.76 vs. $17.78 \pm 5.76 \mathrm{pg} / \mathrm{ml} ; \mathrm{P}=0.132$; Fig. 4B).

Positive correlation between the frequencies of Th22 cells with BNP and CRP in patients with DCM. Finally, the correlation between the percentages of Th22 cells with the levels of laboratory parameters, including BNP, CRP and ESR, were explored. Significantly higher levels of BNP were observed in the DCM group in comparison with the control group 
A

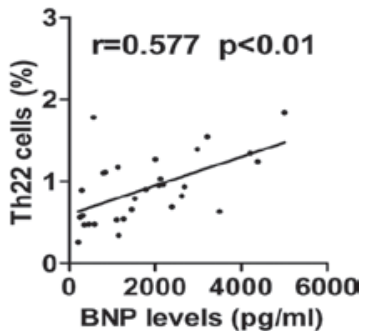

B

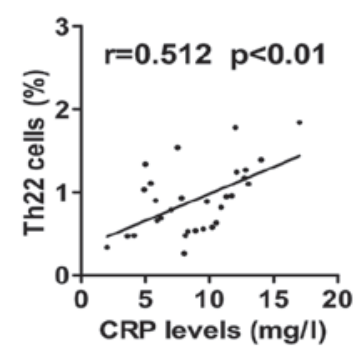

C

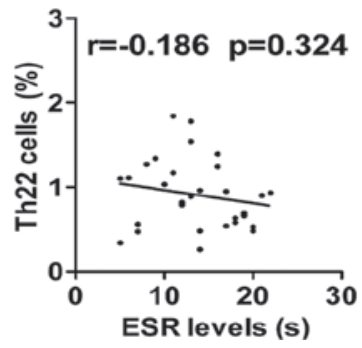

Figure 5. Positive correlation of the frequencies of Th22 cells with BNP, CRP in patients with DCM. (A and B) A significant positive correlation was present between the percentages of peripheral (A) Th22 cells and BNP levels and (B) Th22 cells and CRP in the DCM group. (C) No significant correlation was found between the frequencies of circulating Th22 cells and the values of ESR. Each point represents an individual mouse. Th, T-helper; BNP, brain natriuretic peptide; CRP, C-reactive protein; DCM, dilated cardiomyopathy; ESR, erythrocyte sedimentation rate.

$(1781.9 \pm 1329.8$ vs. $78.6 \pm 21.4 ; \mathrm{P}<0.05)$. As shown in Fig. $5 \mathrm{~A}$, there was a significant positive correlation between proportion of Th22 cells and BNP levels in patients with DCM $(r=0.567$ and $\mathrm{P}<0.01$ ). Additionally, a positive correlation was found between the frequencies of Th22 cells and the expression of CRP ( $\mathrm{r}=0.556$ and $\mathrm{P}<0.01$; Fig. 5B). By contrast, Th22 cells did not show a statistically significant correlation with ESR levels ( $\mathrm{r}=-0.186$ and $\mathrm{P}=0.324$; Fig. 5C).

\section{Discussion}

The identification of Th17 and Th22 cells as distinct subsets of $\mathrm{CD}^{+} \mathrm{T}$ cells has extended the Th1/Th2 paradigm in autoimmune and inflammatory diseases (11-15). However, to date, no studies have directly assessed the presence of circulating Th22 cells in patients with DCM. By applying a multi-parameter cytofluorimetric analysis, the present study revealed that there were significantly increased frequencies of Th22 cells in patients with DCM as compared with healthy controls. In parallel, an elevated expression of AHR, the key transcription factor for Th22-cell differentiation, was observed in the DCM group. These data indicated that an enhanced Th22-cell response may participate in the pathogenesis of DCM. Of note, a previous study by our group reported elevated levels of Th22 cells in viral myocarditis (VMC) (16), a disease which may evolve into DCM $(2,3,17)$. Thus, whether higher levels of Th22 cells are involved in the evolvement from VMC to DCM, is an interesting unsettled question. It should be mentioned that the levels of Th22 cells identified in the present study appear to differ from those in other reports $(13,15)$. It is hypothesized that these discrepancies may be due to different conjugated anti-human-specific antibodies used in the flow cytometric analysis. Furthermore, the enrichment of Th22 cells was closely associated with elevated levels of Th17 cells, indicating a polyfunctional cytokine profile and a potentially synergistic function of $\mathrm{CD}^{+} \mathrm{T}$ cells in patients with DCM.

ANT is an autoantibody against the adenosine diphosphate/adenosine triphosphate carrier. A higher frequency of Th22 cells was found in ANT antibody-positive patients with DCM as compared with those in negative subjects. Given that ANT production may have been induced by a loss of tolerance to cardiac self-antigens and is capable of cross-reacting with subunits of the calcium channel on the cardiac cell membrane, which results in myocardial cytotoxic injury $(18,19)$, the findings of the present study indicated that the Th22 response may be involved in organ-specific autoimmunity and myocardial injury in patients with DCM. In addition, a positive correlation was identified between the proportions of Th22 cells and the values of CRP, a significant inflammatory marker and independent predictor of future cardiovascular events $(20,21)$. Furthermore, there was a positive correlation between the percentages of Th22 cells and the levels of BNPs, which are predominantly secreted by cardiac myocytes in response to stretching forces $(22,23)$. BNPs have been demonstrated to be powerful predictors of mortality and major adverse cardiovascular events in patients with DCM (22-25). Thus, these observations implied that Th22 cells may be one predictor for cardiac events in DCM, but further research with larger subjects and a long-term follow-up are warranted.

IL-22 is the most significant functional cytokine of Th22 cells and positive correlations have been observed between elevated levels of IL-22 and Th22 cells in various types of autoimmune diseases $(11-15,26,27)$. The present study observed higher levels of Th22 cells in patients with DCM, and unexpectedly, the plasma levels of IL-22 were comparable between the DCM and control group. Furthermore, IL-22 levels did not show a statistically significant difference between ANT-positive and -negative patients with DCM. In addition, plasma concentrations of IL-22 did not show a statistically significant correlation with the percentage of Th22 cells, nor did the Th17 and Th1 cells. The results of the present study were discrepant with previous observations $(11-15,26,27)$, which may be due to different biological characteristics among different autoimmune disorders, indicating that Th22 cells are not the major producer of IL-22 in DCM. IL-22 may be produced by various cell types in the microenvironment of DCM, including innate lymphoid cells, natural killer and $\gamma \delta$ T cells, but production is not limited to Th22, Th1 and Th17 cells (28-30).

Principal limitations of the present study are the small sample size, observations made at only one time-point and single blood sampling. Furthermore, due to the limited number of subjects with DCM, a definitive causal correlation between the Th22 cells and New York Heart Association (NYHA) func- 
tional impairment was not identified. In addition, the subjects of the present study were primarily comprised of men; thus, these results may not necessarily apply to women. Further prospective studies enrolling larger cohorts of patients are required to further elucidate the pathophysiological function and prognostic role of Th22 cells in patients with DCM.

In conclusion, the present study demonstrated an elevation of Th22 cells in patients with DCM, particularly in ANT autoantibody-positive subjects. These novel findings imply that the Th22 response may participate in the pathogenesis of DCM. Further studies in a larger sized population are required to validate these findings and to determine the role of Th22 in the immunopathogenesis of DCM.

\section{Acknowledgements}

The present study was supported by the National Natural Science Foundations of China (nos. 81260045 and 81160032). The authors would like to thank Dr Jiao Lan for technical assistance.

\section{References}

1. Richardson P, McKenna W, Bristow M, et al: Report of the 1995 World Health Organisation/International Society and Federation of Cardiology Task Force on the Definition and Classification of Cardiomyopathies. Circulation 93: 841-842, 1996.

2. Luk A, Ahn E, Soor GS and Butany J: Dilated cardiomyopathy: a review. J Clin Pathol 62: 219-225, 2009.

3. Grogan M, Redfield MM, Bailey KR, et al: Long-term outcome of patients with biopsy-proven myocarditis: comparison with idiopathic dilated cardiomyopathy. J Am Coll Cardiol 26: 80-84, 1995.

4. Yuan J, Cao AL, Yu M, et al: Th17 cells facilitate the humoral immune response in patients with acute viral myocarditis. J Clin Immunol 30: 226-234, 2010.

5. Guedes PM, Gutierrez FR, Silva GK, et al: Deficient regulatory $\mathrm{T}$ cell activity and low frequency of IL-17-producing $\mathrm{T}$ cells correlate with the extent of cardiomyopathy in human Chagas disease. PLoS Negl Trop Dis 6: e1630, 2012.

6. Li J, Wang L, Wang S, et al: The Treg/Th17 imbalance in patients with idiopathic dilated cardiomyopathy. Scand J Immunol 71: 298-303, 2010.

7. Yi A, Jian L, Xiaojing H and Hui X: The prevalence of Th17 cells in patients with dilated cardiomyopathy. Clin Invest Med 32: E144-E150, 2009.

8. Okazaki T and Honjo T: Pathogenic roles of cardiac autoantibodies in dilated cardiomyopathy. Trends Mol Med 11: 322-326, 2005.

9. Duhen T, Geiger R, Jarrossay D, Lanzavecchia A and Sallusto F: Production of interleukin 22 but not interleukin 17 by a subset of human skin-homing memory T cells. Nat Immunol 10: 857-863, 2009.

10. Zhang N, Pan HF and Ye DQ: Th22 in inflammatory and autoimmune disease: prospects for therapeutic intervention. Mol Cell Biochem 353: 41-46, 2011.

11. Tian T, Yu S and Ma D: Th22 and related cytokines in inflammatory and autoimmune diseases. Expert Opin Ther Targets 17: 113-125, 2013.
12. Shen H, Goodall JC and Hill Gaston JS: Frequency and phenotype of peripheral blood Th17 cells in ankylosing spondylitis and rheumatoid arthritis. Arthritis Rheum 60: 1647-1656, 2009.

13. Truchetet ME, Brembilla NC, Montanari E, Allanore Y and Chizzolini C: Increased frequency of circulating Th22 in addition to Th17 and Th2 lymphocytes in systemic sclerosis: association with interstitial lung disease. Arthritis Res Ther 13: R166, 2011.

14. Baba N, Rubio M, Kenins L, et al: The aryl hydrocarbon receptor (AhR) ligand VAF347 selectively acts on monocytes and naïve CD4(+) Th cells to promote the development of IL-22-secreting Th cells. Hum Immunol 73: 795-800, 2012.

15. Hu Y, Li H, Zhang L, et al: Elevated profiles of Th22 cells and correlations with Th17 cells in patients with immune thrombocytopenia. Hum Immunol 73: 629-635, 2012.

16. Kong Q, Wu W, Yang F, et al: Increased expressions of IL-22 and Th22 cells in the coxsackievirus B3-induced mice acute viral myocarditis. Virol J 9: 232, 2012.

17. Maron BJ, Towbin JA, Thiene G, et al: Contemporary definitions and classification of the Cardiomyopathies: an American Heart Association Scientific Statement From the Council on Clinical Cardiology, Heart Failure and Transplantation Committee; Quality of Care and Outcomes Research and Functional Genomics and Translational Biology Interdisciplinary Working Groups; and Council on Epidemiology and Prevention. Circulation 113: 1807-1816, 2006.

18. Liao YH: Functional analysis of autoantibodies against ADP/ATP carrier from dilated cardiomyopathy. Int J Cardiol 54: 165-169, 1996.

19. Schulze K, Witzenbichler B, Christmann C and Schultheiss HP: Disturbance of myocardial energy metabolism in experimental virus myocarditis by antibodies against the adenine nucleotide translocator. Cardiovasc Res 44: 91-100, 1999.

20. De Gennaro L, Brunetti ND, Cuculo A, Pellegrino PL and Di Biase M: Systemic inflammation in nonischemic dilated cardiomyopathy. Heart Vessels 23: 445-450, 2008.

21. Kones R: Rosuvastatin, inflammation, C-reactive protein, JUPITER, and primary prevention of cardiovascular disease - a perspective. Drug Des Devel Ther 4: 383-413, 2010.

22. Wang F, Wu Y, Tang L, et al: Brain natriuretic peptide for prediction of mortality in patients with sepsis: a systematic review and meta-analysis. Crit Care 16: R74, 2012.

23. Goetze JP, Christoffersen C, Perko M, Arendrup H, Rehfeld JF, Kastrup J and Nielsen LB: Increased cardiac BNP expression associated with myocardial ischemia. FASEB J 17: 1105-1107, 2003.

24. Nishii $\mathrm{M}$, Inomata $\mathrm{T}$, Takehana $\mathrm{H}$, et al: Prognostic utility of B-type natriuretic peptide assessment in stable low-risk outpatients with nonischemic cardiomyopathy after decompensated heart failure. J Am Coll Cardiol 51: 2329-2335, 2008.

25. Shimazu S, Hirashiki A, Okumura T, et al: Association between indoxyl sulfate and cardiac dysfunction and prognosis in patients with dilated cardiomyopathy. Circ J 77: 390-396, 2013.

26. Peng D, Xu B, Wang Y, Guo H and Jiang Y: A high frequency of circulating Th22 and Th17 cells in patients with new onset graves' disease. PLoS One 8: e68446, 2013.

27. Yang XY, Wang HY, Zhao XY, Wang LJ, Lv QH and Wang QQ: Th22, but not Th17 might be a good index to predict the tissue involvement of systemic lupus erythematosus. J Clin Immunol 33: 767-774, 2013.

28. Wolk K, Kunz S, Asadullah K and Sabat R: Cutting edge: immune cells as sources and targets of the IL-10 family members? J Immunol 168: 5397-5402, 2002.

29. Wolk K, Witte E, Wallace E, et al: IL-22 regulates the expression of genes responsible for antimicrobial defense, cellular differentiation, and mobility in keratinocytes: a potential role in psoriasis. Eur J Immunol 36: 1309-1323, 2006.

30. Simonian PL, Wehrmann F, Roark CL, Born WK, O'Brien RL and Fontenot AP: gammadelta $\mathrm{T}$ cells protect against lung fibrosis via IL-22. J Exp Med 207: 2239-2253, 2010. 